\title{
Design of a New Type Air-to-Ground Power Grid's Hot Spots' Monitoring and Control System
}

\author{
Hongzhao Li, Fumin Yang \\ Beijing Polytechnic, Beijing 100176 \\ hunter2011@foxmail.com
}

Keywords: Air-to-Ground, Integrated Monitoring, Hot Spots in Power Grid

\begin{abstract}
This article designed and realized a new type air-to-ground power grid's hot spots' monitoring and control system, to solve current problems like weak relevance between hot spots in power grid, delay in finding abnormal hot spots, etc. The system is made of long-distance temperature control and monitoring module, master control module, wireless communication module, pan-tilt-device module, etc.; multiple temperature sensors were set up in large power grid regions, and information was gathered to the sub-base station through wireless communication network; when there is an abnormal temperature increase, the pan-tilt-device system of the sub-base station will be used to monitor the region with the hot spot; thus, real time condition of the target region can be monitored effectively, the cost of construction for the monitoring system will also decrease.
\end{abstract}

\section{Introduction}

Abnormal hot spot monitoring in large power grid has been the focus of many scholars' research in military and civil fields, which obtained extremely widespread application[1]. Electric power grid hot spot monitoring with its wide field of vision, the advantages of fast speed, strong adaptability, became key problems in areas such as the fire monitoring and emergency rescue of power grid[2].

To conduct a comprehensive monitoring requires a lot of manpower, financial resources, due to the large monitored area, so, in order to better carry out ground power grid's hot spots' monitoring work, it is a must to build the information transmission analysis network with stronger correlations[3], collect the information in the mission area through a variety of detection means, and monitor the largest area with the smallest investment; sensors interconnect and transmit information among themselves through wireless communication network, and ultimately all information will be collected to monitoring management command center for processing, corresponding control measures will be taken.

\section{System Design}

System Structure. The system is made of temperature sensor module, image detection module, FY-SP2515D pan-tilt-device module, Zigbee wireless communication module, MCU master control module, image display module, sound-light alarm module, power supply, etc.. Temperature sensors are installed at major nodes in the monitored power grid region, they communicate with sub-base station through wireless communication module; infrared thermal imaging system is installed at sub-base station, on the pan-tilt-device; pan-tilt-device can be controlled by the mission area power grid monitor center, the data at sub-base station are processed and collected to monitoring and control management command center[4]. Once the temperature sensors, FY - SP2515D pan-tilt-device module found temperature anomaly in the power grid, the front-end monitoring system will send the alarm information back to the control center, the control center after receiving the alarm information, make an analysis according to the situation, and give orders to the management personnel, to control the temperature anomaly area[5]. The overall structure of the monitoring and control system is shown in figure 1, 


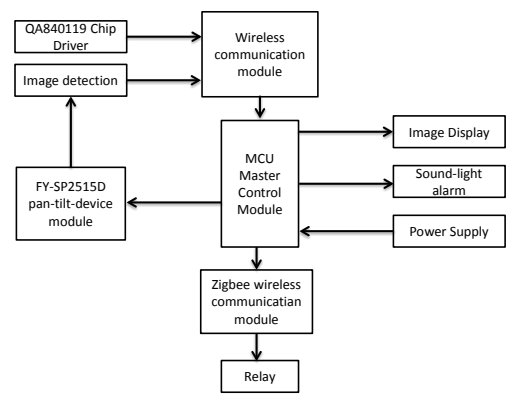

Fig. 1 System's Overall Structure

Hot spots' detection based on FY-SP2515D pan-tilt-device. Sub-base stations were set up in certain regions of the grid, each station set up the infrared thermal imager, installed on the pan-tilt-device, the pan-tilt-device should be at the highest point of the monitored area, the rotation of the pan-tilt-device can be automatically controlled or controlled by the control center. FY SP2515D pan-tilt-device automatically controls itself to rotate 360 degrees for detection, information collected from the monitored grid region were encoded and transmitted through wireless communication network to the hot spots' monitoring center, real-time alarm and monitoring information is displayed real-time at the monitoring center. Once an abnormal temperature is found, control signal can be sent to the front end through the control center, to control FY - SP2515D pan-tilt-device to track target, the control center can make analysis according to the situation, give orders to ground management personnel.

Infrared imaging module has a thermal imager for over-temperature detection and optical detection function; during the daytime when thermal imaging detects high temperature target, visible light cameras detect abnormal hot spot at the same time, pan-tilt-device stops cruise, and send alarm information to the remote monitoring host. At night due to limited environmental disturbance, as long as thermal imaging detects over-temperature target, abnormal condition can be recognized.

Structure Design of System's Key Hardware Module. (1)Hardware Design of MCU Master Control Module. The hardware design structure of MCU master control module of grid abnormal hot spot monitoring system is shown in figure 2, the power supply of the MCU's whole circuit is provided by the $\mathrm{J} 1$ side ac input, the input ac voltage goes through the bridge type full-wave rectifier, filter, and voltage regulator chip 7805, is converted to $+5 \mathrm{~V}$ dc power supply, to be used by the circuit. MCU controls pan-tilt-device's automatic rotation through rotary motor, to make the FY - SP2515D pan-tilt-device collect regional thermal imaging in all directions. Serial port pins TXD, RXD connect to the wireless communication module, send and receive data. P1.5 P1.7 is the serial data interface of the D/A conversion chip .

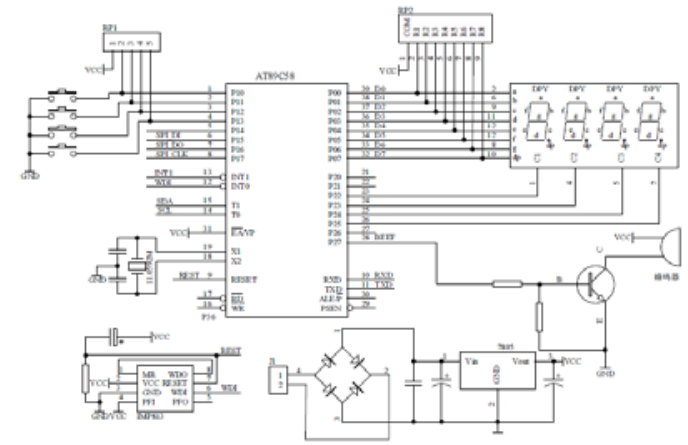

Fig. 2 Schematic of Hardware Design of MCU Master Control Module

(2)Hardware Design of Zigbee wireless communication module. Data from the front-end sensor of Air-to-ground hot spot monitoring and control system transmit information through the Zigbee wireless communication modules, the modules can communicate with each other, a communication network can be formed by means of information transmission, monitoring data is eventually 
gathered to the sub-base station through the wireless sensor network (WSN). Pins AVDD SOC and AVDD RREG connect the analog circuits to the voltages from $2.0 \mathrm{~V}$ to $3.6 \mathrm{~V}$, they respectively connect to $100 \mathrm{nF}$ and $220 \mathrm{nF}$ filtering capacitors. Pin DVDD is an input pin for digital power, provides power for the I/O port, which connects to a $100 \mathrm{nF}$ filter capacitor. Pin DCOUPL provides a decoupling voltage of $1.8 \mathrm{~V}$, not for external use, which should connect to a $220 \mathrm{nF}$ bypass decoupling capacitor. Pin the X1 is externally connected to a $32 \mathrm{MHZ}$ crystal used exclusively for a $2.4 \mathrm{GHz}$ radio frequency circuit, its bypass capacitor is $22 \mathrm{pF}$. Pins RBIASl and RBIAS2 connect to bias resistors, to provide a suitable working current for the $32 \mathrm{M}$ crystal .

(3)Hardware Design of the Temperature Sensing Module. The hardware design circuit of the temperature sensing module is shown in Figure 3, the circuit is driven by the QA840119 chip, the temperature anomaly in the monitoring area is converted into a sampling voltage signal through the temperature sensor, varying in $16 \sim 32 \mathrm{~V}$, current changes in the region of $0.4 \sim 0.5 \mathrm{~mA}$, the measured temperature is $-30 \sim+85{ }^{\circ} \mathrm{C}$. Sampling voltage is input into the ADI port. When the temperature of the detected object changes abnormally, the temperature probe will produce electric potential difference signals in the circuit, the electric potential of the non-inverting terminal of the operational amplifier is higher than that of the inverting terminal, after the signal is amplified by the differential operational amplifier, the output of the differential mode voltage signal will be sent from the ADO terminal into TLC1543 digital-to-analog conversion chip.

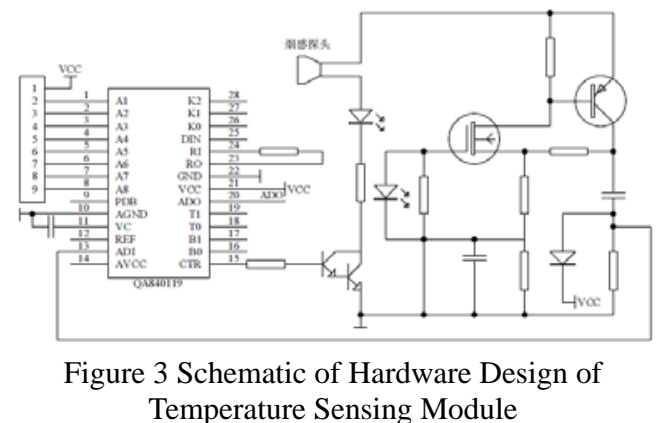

(4)Hardware Design of FY-SP2515D Pan-tilt-device Module. The sensing front end of pan-tilt-device FY - SP2515D of air-to-ground hot spot monitoring system uses the FY-SP2515D pan-tilt-device and optical imaging camera all-in-one machine to function, and communicates data with upper computer through the serial port, pan-tilt-device FY - SP2515D located in the sub-base station detects the object's infrared radiation thermal image in monitored area and display it with grayscale or pseudo color, in this way the temperature distribution of the measured target object is obtained and its state can be decided. Background temperature of the monitored area is commonly $-40 \sim 60$ degrees Celsius, and the temperature at the temperature anomaly point is $200 \sim 1200$ degrees Celsius, the temperature difference is very big. It is easy to separate temperature anomalies from terrain background in the thermal image.

\section{Software Design of Hot Spot Monitoring System}

Software Design of The System's Main Process. The software design of this system is based on C language, when the hot spot monitoring temperature sensor detects object's temperature anomaly, FY - SP2515D pan-tilt-device did signal acquisition, the signal then is sent to the monitoring center through wireless communication module for processing, the system automatically aims the infrared thermal imager at the area which sends out alarms, to do real-time thermal imaging to the monitored area. The system's main process' software design is shown in figure 4. 


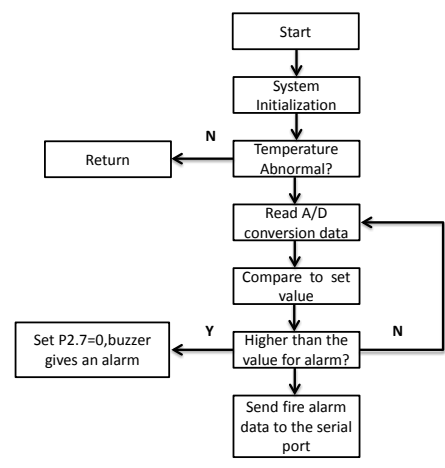

Fig. 4. Schematic of Software Design for System's Main Process

Software Implementation of FY-SP2515D Pan-tilt-device Communication Protocol in the Hot Spot Monitoring System. Working mode of FY-SP2515D Pan-tilt-device's communication port is: N-8-1 format, namely one start bit, 8 data bits, 1 stop bit, no verification; Communication baud rate support from 1200 to 115200 bps. The command structure is: [0xAA] [instruction code] [data] [0xCC 0x33 0xC3 0x3C].

(1)0xAA: Command head, must be sent before sending each instruction, determine the start of a command based on this byte.

(2)Instruction code: different instruction codes represent different meanings, 0x01 (normal), 0x02 (detected smoke), etc.

(3)Data: up to 2000 bytes, the specific definition of each byte in the data varies according to different command codes.

(4)0xCC 0x33 0xC3 0x3C: The end of the code, only to receive these 4 bytes, it is decided that a command is over, and then next step is to translate and to execute the command.

Implementation of Air-to-ground Hot Spot Monitoring System's Filtering Software. Because ambient interference like air flow interferes the sensor's signal acquisition, software is needed to do filtering processing on collected signals, the particular way is to collect the signal at intervals of $100 \mathrm{~ms}$ and store it, in 1 second you'd collect 10 signals, next do sum and arithmetic average on them, this way the data stability of the collected signals can be effectively improved, conditions like the false alarm from the jamming signal can be prevented.

\section{Experiments and Analysis}

Select an empty area to test system performance, connect to PC through wireless communication module, and keep record of the system operation condition and collected experimental data, the result is shown in Table 1.

Table 1 Experimental Data

\begin{tabular}{|l|l|}
\hline \multicolumn{1}{|c|}{ Indicator Content } & \multicolumn{1}{c|}{ Detection Indicator } \\
\hline System running OK & \multicolumn{1}{c|}{ Yes } \\
\hline Abnormal Temperature Detection & $95 \%$ \\
\hline Normal Temperature Detection & $95 \%$ \\
\hline Size of abnormal grid region & $10 \times 10 \times 10$ \\
\hline Buzzer sounds an alarm & Yes \\
\hline
\end{tabular}

Conduct simulation on the data from the grid's hot spot temperature anomaly collected by FY-SP2515D pan-tilt-device using the MATLAB software, the area of the temperature anomaly range in the monitored region and temperature change trend, etc. can be seen from the simulation image.

Through experiments, the result of simulation shows that, the system can effectively detect the grid's temperature's abnormal changes and send out alarm information, wireless communication works fine, FY - SP2515D pan-tilt-device system can accurately detect abnormal changes of hot spots, provide a basis for the analysis of the accident, the whole system's design meet the need for the work of monitoring regional abnormal hot spots. 
Conduct measurement on 10 temperature anomaly simulation spots, do experiments on the operation time, detection error and quantity of energy saving of this system and the traditional system, the experimental results are shown in Table 2 and Fig5, respectively.

Table 2 Experimental Results

\begin{tabular}{|c|c|c|c|c|c|c|}
\hline \multirow{2}{*}{$\begin{array}{l}\text { Number } \\
\text { of spot }\end{array}$} & \multicolumn{2}{|c|}{ Calculation time(s) } & \multicolumn{2}{|c|}{ Detection error $(\%)$} & \multicolumn{2}{|c|}{$\begin{array}{c}\text { Quantity of energy } \\
\text { saving (J) }\end{array}$} \\
\hline & $\begin{array}{c}\text { Traditional } \\
\text { system }\end{array}$ & $\begin{array}{c}\text { Our } \\
\text { system }\end{array}$ & $\begin{array}{c}\text { Traditional } \\
\text { system }\end{array}$ & $\begin{array}{c}\text { Our } \\
\text { system }\end{array}$ & $\begin{array}{c}\text { Traditional } \\
\text { system }\end{array}$ & Our system \\
\hline 1 & 6.5 & 1.2 & 11.8 & 1.3 & 143 & 268 \\
\hline 2 & 5.8 & 1.3 & 5.9 & 1.5 & 139 & 269 \\
\hline 3 & 6.6 & 1.4 & 6.1 & 1.4 & 201 & 261 \\
\hline 4 & 5.9 & 1.6 & 6.0 & 1.6 & 140 & 271 \\
\hline 5 & 5.7 & 1.3 & 5.9 & 1.4 & 203 & 281 \\
\hline 6 & 6.9 & 1.1 & 6.3 & 1.2 & 177 & 266 \\
\hline 7 & 5.6 & 1.7 & 5.8 & 1.4 & 92 & 259 \\
\hline 8 & 5.2 & 1.5 & 5.7 & 1.3 & 107 & 263 \\
\hline 9 & 7.5 & 1.6 & 7.8 & 1.4 & 132 & 289 \\
\hline 10 & 5.4 & 1.5 & 5.8 & 1.3 & 154 & 279 \\
\hline
\end{tabular}

From the experimental results of above Table 2, the quantity of energy saving of the system in this paper saves $120 \mathrm{~J}$ in average compared to that of traditional system, and the computation efficiency of the system in this paper is superior to that of the traditional system, increasing by about $22 \%$, and the detection error of our system is within 2\%. Experimental data shows that the system of this paper can ensure maximum energy saving in temperature anomaly detection, ensure the high work efficiency and accuracy of the detection system , and have important application value.

In order to further validate the performance of system, the system's adaptive degree is experimented, the results are as follows:

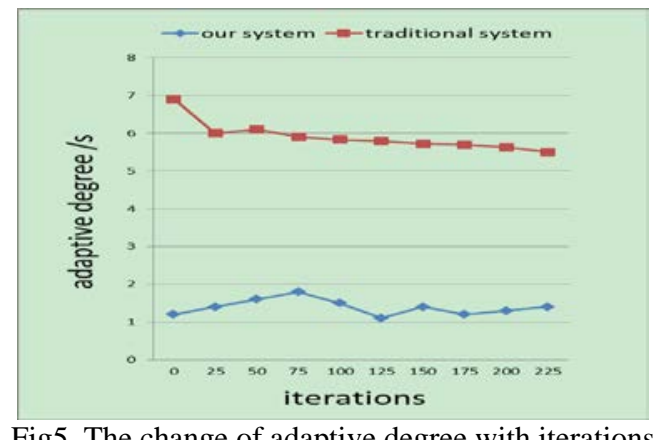

It can be seen from the graph that the best adaptive degree of traditional systems is $6.566 \mathrm{~s}$, and the adaptive degree of the system of this paper is $1.744 \mathrm{~s}$, which improves by $73.4 \%$. This shows that our system has strong adaptivity and is suitable to be popularized extensively.

\section{Summary}

This paper designed and implemented air-to-ground power grid's hot spot monitoring system based on the FY - SP2515D pan-tilt-device, which perfect and upgrade the existing power grid regional temperature anomaly monitoring system, the abnormal change of regional hot spots has a variety of characterization parameters, make a comprehensive judgment on the accidents monitor condition of the monitored area through a variety of means, the data such as the size, location, development trend of the temperature anomaly area can be more accurately determined, this elevates the degree of intellectualization and informatization of power grid's regional hot spot monitoring system, the system's temperature detection sensitivity has increased, the rate of false alarms decreases, the system has a broad application prospect.

\section{Reference}

[1] N. Yang, X.J. Hu; MCU and Control Technology; Beijing: Beijing University of Aeronautics and Astronautics Press, 2005, 13-15.

[2] L. Cui, H.L. Ju, Y. Miao, etc., “Overview of Wireless Sensor Networks”, Journal of Computer Research and Development, 2005, 42(1), 164-165. 
[3] G.F. Li, L.E. Li; MCU C Program Design Example and Guide; Beijing:Beijing University of Aeronautics and Astronautics Press, 2005, 14-15.

[4] Z.P. Zhang, X.Y. Song, Y.F. Li, “Application of Infrared Thermography System in Studying the Cooling Characteristics of Different Vegetables during Vacuum Cooling Process”, BULLETIN OF SCIENCE AND TECHNOLOGY, 2009, 25(3), 295-299.

[5] R. Zhang, S.L. Sun, X.G. Yu; Fundamentals of Embedded System Technology; Beijing: Beijing University of Posts and Telecommunications Press, 2006, 28-29. 